\title{
Correction to: Hematopoietic cell transplantation utilization and outcomes for primary plasma cell leukemia in the current era
}

\author{
Binod Dhakal - Sagar Patel (1) - Saulius Girnius - Lohith Bachegowda - Raphael Fraser - Omar Davila • \\ Abraham S. Kanate - Amer Assal (D) Amr Hanbali - Asad Bashey - Attaphol Pawarode - César O. Freytes • \\ Cindy Lee - David Vesole • Robert Frank Cornell • Gerhard C. Hildebrandt • Hemant S. Murthy • Hillard M. Lazarus • \\ Jan Cerny · Jean A. Yared · Jeffrey Schriber · Jesus Berdeja - Keith Stockerl-Goldstein · Kenneth Meehan • \\ Leona Holmberg • Melhem Solh • Miguel Angel Diaz • Mohamed A. Kharfan-Dabaja (D) Nosha Farhadfar • \\ Qaiser Bashir • Reinhold Munker - Richard F. Olsson - Robert P. Gale • Ruthlee-Lu Bayer • Sachiko Seo • \\ Saurabh Chhabra · Shahrukh Hashmi D - Sherif M. Badawy • Taiga Nishihori (D) Wilson Gonsalves • Yago Nieto • \\ Yvonne Efebera (D) - Shaji Kumar (D) N Nina Shah • Muzaffar Qazilbash (D) Parameswaran Hari • Anita D'Souza
}

Published online: 29 March 2021

(c) The Author(s), under exclusive licence to Springer Nature Limited 2021

Correction to: Leukemia https://doi.org/10.1038/s41375-020-0830-0

The acknowledgement of the original article was incomplete. Correct is should read:

The CIBMTR is supported primarily by Public Health Service U24CA076518 from the National Cancer Institute (NCI), the National Heart, Lung and Blood Institute (NHLBI) and the National Institute of Allergy and Infectious Diseases (NIAID); U24HL138660 from NHLBI and NCI; U24CA233032 from the NCI; OT3HL147741, and U01HL128568 from the NHLBI; HHSH250201700006C, and HHSH250201700007C from the Health Resources and Services Administration (HRSA); and N00014-20-12705 and N00014-20-1-2832 from the Office of Naval Research; Additional federal support is provided by P01CA111412, R01CA152108, R01CA215134, R01C A218285, R01CA231141, R01AI128775, R01HL126589, R01HL129472, R01HL130388, R01HL131731， U01AI 069197, U01AI126612, UG1HL06924, and BARDA. Support is also provided by Be the Match Foundation, Boston Children's Hospital, Dana Farber, St. Baldrick's Foundation,
Stanford University, the Medical College of Wisconsin the National Marrow Donor Program, and from the following commercial entities: Actinium Pharmaceuticals, Inc.; Adienne SA; Allovir, Inc.; Amgen, Inc.; Angiocrine Bioscience; Astellas Pharma US; bluebird bio, Inc.; Bristol Myers Squibb Co.; Celgene Corp.; CSL Behring; CytoSen Therapeutics, Inc.; Daiichi Sankyo Co., Ltd.; ExcellThera; Fate Therapeutics; Gamida-Cell, Ltd.; Genentech Inc; Incyte Corporation; Janssen/Johnson \& Johnson; Jazz Pharmaceuticals, Inc.; Kiadis Pharma; Kite, a Gilead Company; Kyowa Kirin; Legend Biotech; Magenta Therapeutics; Merck Sharp \& Dohme Corp.; Millennium, the Takeda Oncology Co.; Miltenyi Biotec, Inc.; Novartis Pharmaceuticals Corporation; Omeros Corporation; Oncoimmune, Inc.; Orca Biosystems, Inc.; Pfizer, Inc.; Pharmacyclics, LLC; Sanofi Genzyme; Stemcyte; Takeda Pharma; Vor Biopharma; Xenikos BV. The views expressed in this article do not reflect the official policy or position of the National Institute of Health, the Department of the Navy, the Department of Defense, Health Resources and Services Administration (HRSA) or any other agency of the U.S. Government.

The original article has been corrected. 